Editorial

\title{
Impact of Quantitative Pharmacology on Drug Development
}

\author{
Ana Ruiz-Garcia and Kourosh Parivar \\ Clinical Pharmacology, Pfizer Oncology, United States
}

The success of drug development rests over two well defined pillars, a good understanding of the drug's clinical pharmacology and the appropriate target population for whom the clinical use is intended. The label for human prescription drugs requires a good understanding of the pharmacological effects (pharmacodynamics or PD) and the mechanism of action of the drug as well as detailed information of the drug's Pharmacokinetics (PK): Absorption, distribution, metabolism and excretion (FDA, 2014). The understanding of the PK characteristics and PD effects (desired or adverse) will provide educated recommendations about the effective dose, dosing regimen, potential drug-drug interactions and hence contraindications and warnings. Further, the influence of demographic factors on the PK and PD attributes (e.g., age, sex, race, hepatic or renal impairment) need to be well understood to provide the appropriate guidance to patients and caregivers for patients in specific populations (pediatric, geriatric, organ impairment, pregnancy, etc). In summary, a very extensive portion of the label covers clinical pharmacology topics, the following label sections are mainly supported by clinical pharmacology knowledge collected throughout the course of drug development:

- Dosage and administration: Including the dose, frequency of administration and route of administration.

- Clinical Pharmacology:

- Mechanism of Action

- Pharmacodynamics

- Pharmacokinetics (ADME)

- Pharmacogenomics (if appropriate)

- Drug interactions

- Contraindications

- Use in specific populations

The methodology used to support the label claims in the last decade has been strongly influenced from the industry shift towards "learning Vs. confirming" introduced by Lewis (1997). The traditional way of drug development has been much focused on empirical testing of New Chemical Entities (NCE) through classical Phase I, II and III drug development with subsequent high failure rate in Phase III. However, with the learning Vs confirming paradigm the intellectual focus of clinical drug development has been on understanding the PK and PK/PD characteristics of the NCEs during each stage of drug development and using that knowledge in planning the next step. Planning and analyzing has become as important, if not more, than execution. Quantitative pharmacology has clearly emerged as key component of drug development and decision making. Very intuitive and straight forward definitions for 2 of the big aspects of clinical pharmacology, PK and PD were provided by Professor Ritschel (1973), which later (1984) Leslie Benet introduced in English (Jerry and Horning, 1984):

Pharmacokinetics is what the body does to the drug-pharmacodynamics is what the drug does to the body.

Zhang et al. (2008) indicated quantitative Model-Based Drug Development (MBDD) as a fundamental tool to improve efficiency and success rate in drug development. Regulatory agencies including the US Food and Drug Administration (FDA) and European Agency for the Evaluation of Medicinal Products (EMA) have acknowledged and reflected the use of MBDD in a variety of guidances for industry briefly discussed in this editorial: First in Human (FIH) dose selection (FDA, 2005a), exposure-response relationships (FDA, 2003a), QT/QTc (FDA, 2005b), drug interaction studies (FDA, 2012), renal and hepatic impairment studies (FDA, 2010a; EMA, 2014; EMEA, 2005; FDA, 2003b), Endof-Phase 2A (EOP2A) meeting (FDA, 2009), population pharmacokinetics (FDA, 1999; EMEA, 2007) and adaptive design trials (FDA, 2010b).

Both FDA and EMA have stated the importance of MBDD to justify the selection of FIH dose in their guidance indicating that the No Observed Adverse Effect Level (NOAEL) determined in preclinical toxicology studies in the most sensitive and relevant animal species should not be the only approach. Quoting the guidance document:
"Although the process outlined in this document uses observed toxicities, administered doses and an algorithmic approach to calculate the Maximum Recommended Starting Dose (MRSD), an alternative approach could be proposed that places primary emphasis on animal pharmacokinetics and modeling rather than dose". 
The US FDA guidance of exposure-response relationships emphasizes the importance of MBDD in exposure-response studies and suggests that model-based analysis and simulation be applied to analyze exposureresponse data from clinical as well as preclinical studies. $\mathrm{PK} / \mathrm{PD}$ simulation is also suggested as a way of predicting expected relationships between exposure and response in situations where real data is sparse or absent.

Evaluation of drug effects on the standard Electrocardiogram (ECG) intervals and waveforms is considered a fundamental component of the safety database of any new drug application. Analyses of central tendency, categorical analyses of the QT/QTc interval as well as the relationship between drug exposure and QT/QTc interval changes are outlined in the FDA guidance. PK/PD analysis of the relationship between exposure, often represented as $\mathrm{C}_{\max }$ and QT prolongation may provide additional information to assist in the planning and interpretation of studies assessing cardiac repolarization.

Quantitative pharmacology can be valuable in characterizing the clinical impact of known or newly identified interactions and making recommendations for dosage adjustments. Results from population pharmacokinetic analyses could be informative and sometimes conclusive when the clinical studies are adequately designed to detect significant changes in drug exposure due to Drug-Drug Interactions (DDIs). Moreover, it may also help to detect unsuspected DDIs and provide further evidence of the absence of a DDI when this is supported by prior outcome and mechanistic data.

A study in patients with impaired renal or hepatic function to characterize the PK behavior of the study drug is recommended when either renal or hepatic impairment is likely to significantly alter the PK of the drug and/or its active metabolites. Both Guidance's, EMA (2014) and FDA (2010), agreed on the importance of modeling renal and hepatic function and PK parameters with the goal of providing quantitative basis for dosage recommendations.

Wang et al. (2008) FDA associates summarized the experience across 11 pilot EOP2A meetings between 2004 and 2006 before the guidance was issued. These meetings focused on discussing the exposure-response information during early drug development with the objectives of improving the efficiency of Phase 2B and Phase 3 drug development. Models were built based on Phase 1 and Phase 2A data such as dose response, disease conditions, placebo effect and baseline data. The experience of these pilot meetings suggested that if MBDD strategy is embraced in early clinical development, late-stage drug development would become more efficient and there would be fewer disappointments prior to and after drug approval.
Both, EMA and FDA have drafted guidance's on how to present the results of a population PK analysis, including guidance on the content of the analysis plan for the population analysis. The agencies recommend the use of population analysis in drug development, model validation methods and the appropriate documentation to provide in the population report intended for submission.

All MBDD carries a strong statistical component, in fact, in drug development the use of adequate statistical methods is key in designing, conducting, evaluating and interpreting results. Adaptive (flexible) designs are promoted by EMA and FDA. Model based designs use accumulating data to decide on how to modify aspects of the study as it continues, without undermining the validity and integrity of the trial. The main goal is to learn from the accumulating data and to apply what is learned as quickly as possible.

In summary, quantitative pharmacology has been identified by regulatory agencies as a valuable discipline with a profound impact in the efficiency and success rate of drug development programs. Learning and confirming using a mathematical approach with the appropriate statistical rigor enables critical decision making bringing well understood and characterized medicines to patients.

\section{References}

EMA, 2014. Guideline on the evaluation of the pharmacokinetics of medicinal products in patients with decreased renal function. European Medicines Agency, UK.

EMEA, 2005. Guideline on the evaluation of the pharmacokinetics of medicinal products in patients with impaired hepatic function. Committee for Medicinal Products for Human Use (CHMP), European Medicines Agency.

EMEA, 2007. Guideline on reporting the results of population pharmacokinetic analyses. Committee for Medicinal Products for Human Use (CHMP), European Medicines Agency.

FDA, 1999. Population pharmacokinetics. U.S Department of Health and Human Services, Food and Drug Administration.

FDA, 2003a. Exposure-response relationships- study design, data analysis and regulatory applications. U.S Department of Health and Human Services, Food and Drug Administration.

FDA, 2003b. Pharmacokinetics in patients with impaired hepatic function: Study design, data analysis and impact on dosing and labeling. U.S Department of Health and Human Services, Food and Drug Administration.

FDA, 2005a. Guidance for industry estimating the maximum safe starting dose in initial clinical trials for therapeutics in adult healthy volunteers. U.S Department of Health and Human Services, Food and Drug Administration. 
FDA, 2005b. E14 clinical evaluation of QT/QTc interval prolongation and proarrhythmic potential for nonantiarrhythmic drugs. U.S Department of Health and Human Services, Food and Drug Administration

FDA, 2009. End-of-Phase 2A meetings. U.S Department of Health and Human Services, Food and Drug Administration.

FDA, 2010a. Pharmacokinetics in patients with impaired renal function. study design, data analysis and impact on dosing and labeling. U.S Department of Health and Human Services, Food and Drug Administration.

FDA, 2010b. Adaptive design clinical trials for drugs and biologics. U.S Department of Health and Human Services, Food and Drug Administration.

FDA, 2012. Drug interaction studies-study design, data analysis, implications for dosing and labeling recommendations. U.S Department of Health and Human Services, Food and Drug Administration.

FDA, 2014. Clinical pharmacology labeling for human prescription drug and biological products. considerations, content and format. U.S Department of Helath and Human Services, Food and Drug Administration.
Jerry, R.M. and M.G. Horning, 1984. Drug Metabolism and Drug Toxicity. 1st Edn., New York, Raven Press, ISBN-10: 0890049971, pp: 436.

Lewis, B.S., 1997. Learning versus confirming in clinical drug development. Clinical Pharmacol. Therapeutics, 61: 275-291. DOI: 10.1016/S0009-9236(97)90160-0

Ritschel, W.A., 1973. Angewandte Biopharmazie. 1st Edn., Germany.

Wang, Y., A.V. Bhattaram, P.R. Jadhav, L.J. Lesko and R. Madabushi et al., 2008. Leveraging prior quantitative knowledge to guide drug development decisions and regulatory science recommendations: Impact of FDA pharmacometrics during 2004-2006. J. Clinical Pharmacol., 48: 146-156. DOI: $10.1177 / 0091270007311111$

Zhang, L., M. Pfister and M. Bernd, 2008. Concepts and challenges in quantitative pharmacology and modelbased drug development. AAPS J., 10: 552-559. DOI: $10.1208 / \mathrm{s} 12248-008-9062-3$ 